\title{
ASYMPTOTIC APPROXIMATIONS OF THE STABLE AND \\ UNSTABLE MANIFOLD OF THE FIXED POINT OF A CERTAIN RATIONAL MAP BY USING FUNCTIONAL EQUATIONS
}

\author{
M.R.S. KULENOVIĆ AND E. PILAV
}

Dedicated to the memory of Professor Mahmut Bajraktarević

\begin{abstract}
We find an asymptotic approximations of the stable and unstable manifolds of the saddle equilibrium solution and the periodtwo solutions of the following difference equation $x_{n+1}=p+x_{n-1} / x_{n}$, where the parameter $p$ is positive number and the initial conditions $x_{-1}$ and $x_{0}$ are positive numbers. These manifolds, which satisfy the standard functional equations of stable and unstable manifolds determine completely global dynamics of this equation.
\end{abstract}

\section{INTRODUCTION}

In this paper we consider the difference equation

$$
x_{n+1}=p+\frac{x_{n-1}}{x_{n}}, \quad n=0,1, \ldots,
$$

where the parameter $p$ is a positive number and the initial conditions $x_{-1}$ and $x_{0}$ are positive numbers. Equation (1) was first considered in [1], where the first trichotomy result was obtained, which was refined in [10]. Neither of these results was global as they did not describe global dynamics in the non-hyperbolic case $p=1$ and in the case $p<1$ when there are unbounded solutions. The following global bifurcation result for Equation (1) was obtained in [6]:

Theorem 1. Equation (1) exhibits the following global behavior:

2010 Mathematics Subject Classification. Primary: 39A10, 39A20; Secondary: 37E99, $37 \mathrm{D} 10$.

Key words and phrases. Basin of attraction, competitive map, functional equation, monotonicity, stable manifold, unstable manifold.

Copyright (c) 2016 by ANUBIH. 
(a) $p<1 \Rightarrow$ every solution off the global stable manifold of the equilibrium is unbounded;

(b) $p=1 \Rightarrow$ every solution converges to a period-two solution;

(c) $p>1 \Rightarrow$ every solution converges to the equilibrium.

In addition, in case (b) there is an infinite number of period-two solutions which are non-hyperbolic and each has its basin of attraction, which is the stable manifold of this solution. This basin of attraction is the graph of a continuous and non-decreasing function and the corresponding period-two solution depends continuously on the initial point.

As it was shown in $[1,10]$ Equation (1) has a unique equilibrium point $\bar{x}=p+1$, which is locally asymptotically stable if $p>1$, a saddle point if $p<1$ and non-hyperbolic of stable type if $p=1$, in which case Equation (1) has an infinite number of period-two solutions which are non-hyperbolic. Furthermore, as a consequence of our results in [14, 15] every unbounded solution in case (a) of Theorem 1 is asymptotic to the unstable manifold of the equilibrium saddle point. Thus to complete the global dynamics of Equation (1) one need to find equations of stable and unstable manifolds of the equilibrium solution and the stable manifold of period-two solutions of Equation (1). In general it is sometimes possible to find the exact equations of these manifolds such as in [4], but most of the times only asymptotic expansions are possible $[2,8]$. It is interesting to notice that it was shown in $[18,20]$ that the stable manifold of the equilibrium solution of Equation (1) is not the graph of any rational function. The asymptotic approximations of stable and unstable manifolds are obtained from the corresponding functional equations which are satisfied by these manifolds, see $[7,9,16,19]$. The advantage of the process of finding the asymptotic approximations of stable and unstable manifolds for Equation (1) lies in the fact that these manifolds are known to be the graphs of continuous and monotonic functions, see $[12,14,15]$ unlike such manifolds for general difference equations such as Henon difference equation which is fractal $[7,9,16,19]$.

The next section gives preliminary results from $[12,14,15]$, which describe global stable and unstable manifolds of competitive maps and global dynamics of such maps as well as connections between such maps and second order difference equations where the transition function decreases in $x_{n}$ and increases in $x_{n-1}$. The final section implements the method of undetermined coefficients in determining the asymptotic approximations of the stable and unstable manifolds of the saddle equilibrium solution and the period-two solutions of Equation (1) and gives some numeric examples and visual comparison with the basins of attraction obtained by another method. 


\section{Preliminaries}

In this section we present some basic results for the competitive maps which describe the existence and the properties of their invariant manifolds.

A first order system of difference equations

$$
\left\{\begin{array}{l}
x_{n+1}=f\left(x_{n}, y_{n}\right) \\
y_{n+1}=g\left(x_{n}, y_{n}\right)
\end{array} \quad, \quad n=0,1, \ldots\right.
$$

where $\mathcal{S} \subset \mathbb{R}^{2},(f, g): \mathcal{S} \rightarrow \mathcal{S}, f, g$ are continuous functions is competitive if $f(x, y)$ is non-decreasing in $x$ and non-increasing in $y$, and $g(x, y)$ is nonincreasing in $x$ and non-decreasing in $y$. If both $f$ and $g$ are nondecreasing in $x$ and $y$, the system (2) is cooperative. Competitive and cooperative maps are defined similarly. Strongly competitive systems of difference equations or strongly competitive maps are those for which the functions $f$ and $g$ are coordinate-wise strictly monotone.

Competitive and cooperative systems have been investigated by many authors, see $[11,12,14,15,17]$. Special attention to discrete competitive and cooperative systems in the plane was given in $[6,12,13,14,15,17]$. One of the reasons for paying special attention to two-dimensional discrete competitive and cooperative systems is the fact that many examples of mathematical models in biology and economy which involve competition or cooperation are models which involve two species. Another reason is that the theory of two-dimensional discrete competitive and cooperative systems is very well developed, unlike such theory for three and higher dimensional systems [17], where some examples of competitive systems with simple dynamics as well as those that exhibit chaos are provided.

If $\mathbf{v}=(u, v) \in \mathbb{R}^{2}$, we denote with $\mathcal{Q}_{\ell}(\mathbf{v}), \ell \in\{1,2,3,4\}$, the four quadrants in $\mathbb{R}^{2}$ relative to $\mathbf{v}$, i.e., $\mathcal{Q}_{1}(\mathbf{v})=\left\{(x, y) \in \mathbb{R}^{2}: x \geq u, y \geq v\right\}$, $\mathcal{Q}_{2}(\mathbf{v})=\left\{(x, y) \in \mathbb{R}^{2}: x \leq u, y \geq v\right\}$, and so on. Define the SouthEast partial order $\preceq_{s e}$ on $\mathbb{R}^{2}$ by $(x, y) \preceq_{s e}(s, t)$ if and only if $x \leq s$ and $y \geq t$. Similarly, we define the North-East partial order $\preceq_{n e}$ on $\mathbb{R}^{2}$ by $(x, y) \preceq_{n e}(s, t)$ if and only if $x \leq s$ and $y \leq t$. For $\mathcal{A} \subset \mathbb{R}^{2}$ and $\mathrm{x} \in \mathbb{R}^{2}$, define the distance from $\mathrm{x}$ to $\mathcal{A}$ as $\operatorname{dist}(\mathrm{x}, \mathcal{A}):=\inf \{\|\mathrm{x}-\mathrm{y}\|: \mathrm{y} \in \mathcal{A}\}$. By $\operatorname{int} \mathcal{A}$ we denote the interior of a set $\mathcal{A}$.

It is easy to show that a map $F$ is competitive if it is non-decreasing with respect to the South-East partial order, that is if the following holds:

$$
\left(\begin{array}{l}
x^{1} \\
y^{1}
\end{array}\right) \preceq_{s e}\left(\begin{array}{c}
x^{2} \\
y^{2}
\end{array}\right) \Rightarrow F\left(\begin{array}{l}
x^{1} \\
y^{1}
\end{array}\right) \preceq_{s e} F\left(\begin{array}{l}
x^{2} \\
y^{2}
\end{array}\right) .
$$

The competitive map $F$ is said to satisfy $(O+)$ condition if $F\left(x_{1}, y_{1}\right) \preceq_{n e}$ $F\left(x_{2}, y_{2}\right)$ implies $\left(x_{1}, y_{1}\right) \preceq_{n e}\left(x_{2}, y_{2}\right)$. The importance of $(O+)$ condition 
lies in the fact that any bounded orbit of a competitive map which satisfy $(O+)$ converges to an equilibrium, see $[12,14,15,17]$.

For standard definitions of attracting fixed point, saddle point, stable and unstable manifold, and related notions see $[9,11,16]$.

The following four results were proved by Kulenović and Merino [15] for competitive systems in the plane, when one of the eigenvalues of the linearized system at an equilibrium (hyperbolic or non-hyperbolic) is by absolute value smaller than 1 while the other has an arbitrary value.

Theorem 2. Let $T$ be a competitive map on a rectangular region (the cartesian product of two intervals in $\mathbb{R}) \mathcal{R} \subset \mathbb{R}^{2}$. Let $\overline{\mathrm{x}} \in \mathcal{R}$ be a fixed point of $T$ such that $\Delta:=\mathcal{R} \cap \operatorname{int}\left(\mathcal{Q}_{1}(\overline{\mathrm{x}}) \cup \mathcal{Q}_{3}(\overline{\mathrm{x}})\right)$ is nonempty (i.e., $\overline{\mathrm{x}}$ is not the $N W$ or $S E$ vertex of $\mathcal{R})$, and $T$ is strongly competitive on $\Delta$. Suppose that the following statements are true.

a. The map $T$ has a $C^{1}$ extension to a neighborhood of $\overline{\mathrm{x}}$.

b. The Jacobian $J_{T}(\overline{\mathrm{x}})$ of $T$ at $\overline{\mathrm{x}}$ has real eigenvalues $\lambda$, $\mu$ such that $0<|\lambda|<\mu$, where $|\lambda|<1$, and the eigenspace $E^{\lambda}$ associated with $\lambda$ is not a coordinate axis.

Then there exists a curve $\mathcal{C} \subset \mathcal{R}$ through $\overline{\mathrm{x}}$ that is invariant and a subset of the basin of attraction of $\overline{\mathrm{x}}$, such that $\mathcal{C}$ is tangential to the eigenspace $E^{\lambda}$ at $\overline{\mathrm{x}}$, and $\mathcal{C}$ is the graph of a strictly increasing continuous function of the first coordinate on an interval. Any endpoints of $\mathcal{C}$ in the interior of $\mathcal{R}$ are either fixed points or minimal period-two points. In the latter case, the set of endpoints of $\mathcal{C}$ is a minimal period-two orbit of $T$.

The following result gives a sufficient condition for the case where the endpoints of $\mathcal{C}$ are boundary points of $\mathcal{R}$.

Theorem 3. For the curve $\mathcal{C}$ of Theorem 2 to have endpoints in $\partial \mathcal{R}$, it is sufficient that at least one of the following conditions is satisfied.

i. The map $T$ has no fixed points nor periodic points of minimal periodtwo in $\Delta$.

ii. The map $T$ has no fixed points in $\Delta$, $\operatorname{det} J_{T}(\overline{\mathrm{x}})>0$, and $T(\mathrm{x})=\overline{\mathrm{x}}$ has no solutions $\mathrm{x} \in \Delta$.

iii. The map $T$ has no points of minimal period-two in $\Delta$, det $J_{T}(\overline{\mathrm{x}})<0$, and $T(\mathrm{x})=\overline{\mathrm{x}}$ has no solutions $\mathrm{x} \in \Delta$.

Theorem 4. (A) Assume the hypotheses of Theorem 2, and let $\mathcal{C}$ be the curve whose existence is guaranteed by Theorem 2 . If the endpoints of $\mathcal{C}$ belong to $\partial \mathcal{R}$, then $\mathcal{C}$ separates $\mathcal{R}$ into two connected components, namely

$$
\begin{aligned}
& \mathcal{W}_{-}:=\left\{\mathrm{x} \in \mathcal{R} \backslash \mathcal{C}: \exists \mathrm{y} \in \mathcal{C} \text { with } \mathrm{x} \preceq_{\text {se }} \mathrm{y}\right\} \text { and } \\
& \qquad \mathcal{W}_{+}:=\left\{\mathrm{x} \in \mathcal{R} \backslash \mathcal{C}: \exists \mathrm{y} \in \mathcal{C} \text { with } \mathrm{y} \preceq_{\text {se }} \mathrm{x}\right\}
\end{aligned}
$$


such that the following statements are true.

(i) $\mathcal{W}_{-}$is invariant, and $\operatorname{dist}\left(T^{n}(\mathrm{x}), \mathcal{Q}_{2}(\overline{\mathrm{x}})\right) \rightarrow 0$ as $n \rightarrow \infty$ for every $\mathrm{x} \in \mathcal{W}_{-}$

(ii) $\mathcal{W}_{+}$is invariant, and $\operatorname{dist}\left(T^{n}(\mathrm{x}), \mathcal{Q}_{4}(\overline{\mathrm{x}})\right) \rightarrow 0$ as $n \rightarrow \infty$ for every $\mathrm{x} \in \mathcal{W}_{+}$.

(B) If, in addition to the hypotheses of part (A), $\overline{\mathrm{x}}$ is an interior point of $\mathcal{R}$ and $T$ is $C^{2}$ and strongly competitive in a neighborhood of $\overline{\mathrm{x}}$, then $T$ has no periodic points in the boundary of $\mathcal{Q}_{1}(\overline{\mathrm{x}}) \cup \mathcal{Q}_{3}(\overline{\mathrm{x}})$ except for $\overline{\mathrm{x}}$, and the following statements are true.

(iii) For every $\mathrm{x} \in \mathcal{W}_{-}$there exists $n_{0} \in \mathbb{N}$ such that $T^{n}(\mathrm{x}) \in \operatorname{int} \mathcal{Q}_{2}(\overline{\mathrm{x}})$ for $n \geq n_{0}$.

(iv) For every $\mathrm{x} \in \mathcal{W}_{+}$there exists $n_{0} \in \mathbb{N}$ such that $T^{n}(\mathrm{x}) \in \operatorname{int} \mathcal{Q}_{4}(\overline{\mathrm{x}})$ for $n \geq n_{0}$.

If $T$ is a map on a set $\mathcal{R}$ and if $\overline{\mathrm{x}}$ is a fixed point of $T$, the stable set $\mathcal{W}^{s}(\overline{\mathrm{x}})$ of $\overline{\mathrm{x}}$ is the set $\left\{x \in \mathcal{R}: T^{n}(\mathrm{x}) \rightarrow \overline{\mathrm{x}}\right\}$ and the unstable set $\mathcal{W}^{u}(\overline{\mathrm{x}})$ of $\overline{\mathrm{x}}$ is the set

$$
\begin{aligned}
\left\{\mathrm{x} \in \mathcal{R} \text { : there exists }\left\{\mathrm{x}_{n}\right\}_{n=-\infty}^{0} \subset \mathcal{R}\right. \text { s.t. } & T\left(\mathrm{x}_{n}\right)=\mathrm{x}_{n+1}, \\
& \left.\mathrm{x}_{0}=\mathrm{x}, \text { and } \lim _{n \rightarrow-\infty} \mathrm{x}_{n}=\overline{\mathrm{x}}\right\} .
\end{aligned}
$$

When $T$ is non-invertible, the set $\mathcal{W}^{s}(\overline{\mathrm{x}})$ may not be connected and made up of infinitely many curves, or $\mathcal{W}^{u}(\overline{\mathrm{x}})$ may not be a manifold. The following result gives a description of the stable and unstable sets of a saddle point of a competitive map. If the map is a diffeomorphism on $\mathcal{R}$, the sets $\mathcal{W}^{s}(\overline{\mathrm{x}})$ and $\mathcal{W}^{u}(\bar{x})$ are the stable and unstable manifolds of $\bar{x}$.

Theorem 5. In addition to the hypotheses of part (B) of Theorem 4, suppose that $\mu>1$ and that the eigenspace $E^{\mu}$ associated with $\mu$ is not a coordinate axis. If the curve $\mathcal{C}$ of Theorem 2 has endpoints in $\partial \mathcal{R}$, then $\mathcal{C}$ is the stable set $\mathcal{W}^{s}(\overline{\mathrm{x}})$ of $\overline{\mathrm{x}}$, and the unstable set $\mathcal{W}^{u}(\overline{\mathrm{x}})$ of $\bar{x}$ is a curve in $\mathcal{R}$ that is tangential to $E^{\mu}$ at $\overline{\mathrm{x}}$ and such that it is the graph of a strictly decreasing function of the first coordinate on an interval. Any endpoints of $\mathcal{W}^{u}(\overline{\mathrm{x}})$ in $\mathcal{R}$ are fixed points of $T$.

Remark 1. Theorems 2, 3, 4 and 5 show that the stable and unstable manifolds of competitive maps, that satisfy certain conditions, are simple monotonic curves which are as smooth as the functions of the map. As is well-known the stable and unstable manifolds of general maps can have complicated structures consisting of many branches or being strange attractors, see $[7,9,19]$ for some examples of polynomial maps such as Henon with 
unstable manifold being a strange attractor. Finally, see [17] for examples of competitive and so cooperative maps in the plane with chaotic attractors.

Remark 2. Let $I$ be an interval of real numbers and let $f \in C^{1}[I \times I, I]$ be such that $f(I \times I) \subseteq \mathcal{K}$ where $\mathcal{K} \subseteq I$ is a compact set. Let $\bar{x} \in I$, be an equilibrium solution of the difference equation

$$
x_{n+1}=f\left(x_{n}, x_{n-1}\right), \quad n=0,1, \ldots
$$

where $f$ is a continuous function which is non-increasing in first variable and non-decreasing in second variable.

Set $x_{n-1}=u_{n}$ and $x_{n}=v_{n}$ in Equation (5) to obtain the equivalent system

$$
\begin{aligned}
& u_{n+1}=v_{n} \\
& v_{n+1}=f\left(v_{n}, u_{n}\right) \quad, \quad n=0,1, \ldots .
\end{aligned}
$$

Let $\mathcal{T}(u, v)=(v, f(v, u))$. Then $\mathcal{T}$ maps $I \times I$ into itself and the second iterate $\mathcal{F}:=\mathcal{T}^{2}$ given by

$$
\mathcal{F}(u, v)=(f(v, u), f(f(v, u), v))
$$

is clearly strictly competitive on $\mathcal{I} \times \mathcal{I}$ [6]. In addition, as it was shown in [13] the map $\mathcal{F}$ satisfies the $(O+)$ condition and so every solution $\left\{x_{n}\right\}$ of Equation (5) has two subsequences $\left\{x_{2 n}\right\}$ and $\left\{x_{2 n+1}\right\}$, which are monotonic, and so every solution of Equation (5) is asymptotic to an equilibrium solution or period-two solution or to the finite or infinite point on the boundary of the region, see [3] for the direct proof. Now Theorems $2-5$ can be applied to Equation (5) to obtain the global stable and unstable manifolds of both equilibrium solutions and period-two solutions as we did in [2]. See [5] for similar results when $f$ in Equation (5) increases in both variables.

\section{InVARIANT MANIFOLDS AND NORMAL FormS}

In this section we obtain the functional equations satisfied by stable and unstable manifolds of the equilibrium solutions of Equation (1) by using the standard method of bringing this equation to the normal form $[7,9,16,19]$.

Let

$$
\left(\begin{array}{l}
\xi_{n+1} \\
\eta_{n+1}
\end{array}\right)=\left(\begin{array}{cc}
\mu_{1} & 0 \\
0 & \mu_{2}
\end{array}\right)\left(\begin{array}{l}
\xi_{n} \\
\eta_{n}
\end{array}\right)+\left(\begin{array}{l}
g_{1}\left(\xi_{n}, \eta_{n}\right) \\
g_{2}\left(\xi_{n}, \eta_{n}\right)
\end{array}\right)
$$

be the system of difference equations in the normal form, where

$$
g_{1}(0,0)=0, \quad g_{2}(0,0)=0, \quad D g_{1}(0,0)=0 \text { and } D g_{2}(0,0)=0 \text {. }
$$

Suppose that $\left|\mu_{1}\right|>1$ and $\left|\mu_{2}\right|<1$. Then, there are two unique invariant manifolds $\mathcal{W}^{u}$ and $\mathcal{W}^{s}$ tangential to $(1,0)$ and $(0,1)$ at $(0,0)$, which are graphs of the maps

$$
\varphi: E_{1} \rightarrow E_{2} \text { and } \psi: E_{1} \rightarrow E_{2}
$$


such that

$$
\varphi(0)=\psi(0)=0 \text { and } \varphi^{\prime}(0)=\psi^{\prime}(0)=0 .
$$

See $[8,9,19]$. Letting $\eta_{n}=\varphi\left(\xi_{n}\right)$ yields

$$
\eta_{n+1}=\varphi\left(\xi_{n+1}\right)=\varphi\left(\mu_{1} \xi_{n}+g_{1}\left(\xi_{n}, \varphi\left(\xi_{n}\right)\right)\right) .
$$

On the other hand by (6)

$$
\eta_{n+1}=\mu_{2} \varphi\left(\xi_{n}\right)+g_{2}\left(\xi_{n}, \varphi\left(\xi_{n}\right)\right) .
$$

Equating these two equations yields

$$
\varphi\left(\mu_{1} \xi_{n}+g_{1}\left(\xi_{n}, \varphi\left(\xi_{n}\right)\right)\right)=\mu_{2} \varphi\left(\xi_{n}\right)+g_{2}\left(\xi_{n}, \varphi\left(\xi_{n}\right)\right) .
$$

Similarly, letting $\xi_{n}=\psi\left(\eta_{n}\right)$ yields

By using (6) we obtain

$$
\xi_{n+1}=\psi\left(\eta_{n+1}\right)=\psi\left(\mu_{2} \eta_{n}+g_{2}\left(\psi\left(\eta_{n}\right), \eta_{n}\right)\right) .
$$

$$
\xi_{n+1}=\mu_{1} \psi\left(\eta_{n}\right)+g_{1}\left(\psi\left(\eta_{n}\right), \eta_{n}\right) .
$$

Equating these two equations yields

$$
\psi\left(\mu_{2} \eta_{n}+g_{2}\left(\psi\left(\eta_{n}\right), \eta_{n}\right)\right)=\mu_{1} \psi\left(\eta_{n}\right)+g_{1}\left(\psi\left(\eta_{n}\right), \eta_{n}\right) .
$$

Thus the functional equations (7) and (8), define the local unstable manifold

$$
\mathcal{W}_{\text {loc }}^{u}=\left\{(\xi, \eta) \in \mathbb{R}^{2}: \eta=\varphi(\xi)\right\},
$$

and the local stable manifold

$$
\mathcal{W}_{\text {loc }}^{s}=\left\{(\xi, \eta) \in \mathbb{R}^{2}: \xi=\psi(\eta)\right\} .
$$

Without loss generality, we can assume that solutions of the functional equations (7) and (8) take the forms

$$
\varphi(\xi)=\alpha \xi^{2}+\beta \xi^{3}+O\left(|\xi|^{4}\right)
$$

and

$$
\psi(\eta)=\gamma \eta^{2}+\delta \eta^{3}+O\left(|\eta|^{4}\right)
$$

Suppose that $\left|\mu_{1}\right|<1$ and $\mu_{2}=1$. Then there exists invariant curve $\mathcal{C}$ which is tangent to $(1,0)$ at $(0,0)$ and it may be represented locally as the graph of a function $h: \mathbb{R} \rightarrow \mathbb{R}$ such that

$$
\begin{aligned}
\mathcal{C}_{l o c}=\left\{(\xi, \eta) \in \mathbb{R}^{2} \mid \eta=h(\xi), h(0)=0\right. & , D h(0)=0, \\
& |\xi|<\delta \text { for a sufficiently small } \delta\} .
\end{aligned}
$$

Just as in the previous case, one can show that for $\eta=h(\xi)$ the following holds:

$$
h\left(\mu_{1} \xi_{n}+g_{1}\left(\xi_{n}, h\left(\xi_{n}\right)\right)\right)=h\left(\xi_{n}\right)+g_{2}\left(\xi_{n}, h\left(\xi_{n}\right)\right) .
$$

Set

$$
u_{n}=x_{n-1} \text { and } v_{n}=x_{n} \text { for } n=0,1, \ldots
$$


and write Equation (1) in the equivalent form:

$$
\begin{aligned}
& u_{n+1}=v_{n} \\
& v_{n+1}=p+\frac{u_{n}}{v_{n}} .
\end{aligned}
$$

Let $T$ be the corresponding map defined by:

$$
T\left(\begin{array}{l}
u \\
v
\end{array}\right)=\left(\begin{array}{c}
v \\
p+\frac{u}{v}
\end{array}\right) \text {. }
$$

It is easy to see that

$$
T^{2}\left(\begin{array}{l}
u \\
v
\end{array}\right)=T\left(T\left(\begin{array}{l}
u \\
v
\end{array}\right)\right)=\left(\begin{array}{c}
\frac{u}{v}+p \\
\frac{v}{v}+p \\
\frac{u}{v}+p
\end{array}\right) .
$$

Then

$$
\left(\begin{array}{l}
u_{n+1} \\
v_{n+1}
\end{array}\right)=T^{2}\left(\begin{array}{l}
u_{n} \\
v_{n}
\end{array}\right) \text { and }\left(\begin{array}{l}
u_{2 n+2} \\
v_{2 n+2}
\end{array}\right)=T^{2}\left(\begin{array}{l}
u_{2 n} \\
v_{2 n}
\end{array}\right)
$$

3.1. Normal form of the map $T$ at the saddle point $\bar{x}$. First, we transform the equilibrium point $(\bar{x}, \bar{x})=(p+1, p+1)$ of $(12)$ to the origin by the translation

$$
\tilde{u}=u-p-1 \text { and } \tilde{v}=v-p-1
$$

under which the corresponding map (12) becomes

$$
\left(\begin{array}{c}
\tilde{u} \\
\tilde{v}
\end{array}\right) \rightarrow F\left(\begin{array}{l}
\tilde{u} \\
\tilde{v}
\end{array}\right)=T\left(\begin{array}{l}
\tilde{u}+p+1 \\
\tilde{v}+p+1
\end{array}\right)-\left(\begin{array}{l}
p+1 \\
p+1
\end{array}\right)=\left(\begin{array}{c}
\tilde{v} \\
\frac{\tilde{u}+p+1}{\tilde{v}+p+1}-1
\end{array}\right) .
$$

Then $F$ has the fixed point $(0,0)$, which corresponds to the fixed point $(\bar{x}, \bar{x})$ of the map $T$. The Jacobian matrix of $F$ at $(0,0)$, has the form

$$
J_{0}=\operatorname{Jac}_{F}(0,0)=\left(\begin{array}{cc}
0 & 1 \\
\frac{1}{p+1} & -\frac{1}{p+1}
\end{array}\right) .
$$

The eigenvalues of (15) are $\mu_{1,2}$ where

$$
\mu_{1}=\frac{2}{1-\sqrt{4 p+5}} \text { and } \mu_{2}=\frac{2}{1+\sqrt{4 p+5}},
$$

and the corresponding eigenvectors are given by

$$
\mathbf{v}_{1}=\left(\frac{1}{2}(1-\sqrt{4 p+5}), 1\right)^{T} \text { and } \mathbf{v}_{2}=\left(\frac{1}{2}(\sqrt{4 p+5}+1), 1\right)^{T}
$$

respectively. 
Then we have that

$$
\begin{aligned}
F\left(\begin{array}{l}
\tilde{u} \\
\tilde{v}
\end{array}\right)=\left(\begin{array}{cc}
0 & 1 \\
\frac{1}{p+1} & -\frac{1}{p+1}
\end{array}\right)\left(\begin{array}{l}
\tilde{u} \\
\tilde{v}
\end{array}\right)+H_{1}\left(\begin{array}{l}
\tilde{u} \\
\tilde{v}
\end{array}\right) \\
\text { where } H_{1}\left(\begin{array}{l}
\tilde{u} \\
\tilde{v}
\end{array}\right):=\left(\begin{array}{c}
0 \\
\frac{\tilde{v}(\tilde{v}-\tilde{u})}{(p+1)(p+\tilde{v}+1)}
\end{array}\right) .
\end{aligned}
$$

Thus, the system (11) is equivalent to

$$
\left(\begin{array}{l}
u_{n+1} \\
v_{n+1}
\end{array}\right)=\left(\begin{array}{cc}
0 & 1 \\
\frac{1}{p+1} & -\frac{1}{p+1}
\end{array}\right)\left(\begin{array}{l}
u_{n} \\
v_{n}
\end{array}\right)+H_{1}\left(\begin{array}{l}
u_{n} \\
v_{n}
\end{array}\right) .
$$

Let

$$
\begin{aligned}
\left(\begin{array}{l}
u_{n} \\
v_{n}
\end{array}\right)=P \cdot\left(\begin{array}{l}
\xi_{n} \\
\eta_{n}
\end{array}\right) \text { where } P & =\left(\mathbf{v}_{1}, \mathbf{v}_{2}\right) \\
& =\left(\begin{array}{cc}
\frac{1}{2}(1-\sqrt{4 p+5}) & \frac{1}{2}(\sqrt{4 p+5}+1) \\
1 & 1
\end{array}\right) .
\end{aligned}
$$

Then system (17) is equivalent to its normal form

$$
\left(\begin{array}{l}
\xi_{n+1} \\
\eta_{n+1}
\end{array}\right)=\left(\begin{array}{cc}
\mu_{1} & 0 \\
0 & \mu_{2}
\end{array}\right)\left(\begin{array}{l}
\xi_{n} \\
\eta_{n}
\end{array}\right)+\left(\begin{array}{c}
\tilde{f}_{1}\left(\xi_{n}, \eta_{n}\right) \\
\tilde{g}_{1}\left(\xi_{n}, \eta_{n}\right)
\end{array}\right),
$$

where

$$
\left(\begin{array}{c}
\tilde{f}_{1}(u, v) \\
\tilde{g}_{1}(u, v)
\end{array}\right):=P^{-1} \cdot H_{1}\left(P \cdot\left(\begin{array}{c}
u \\
v
\end{array}\right)\right) .
$$

By straightforward calculation we obtain that

$$
\begin{gathered}
\tilde{f}_{1}(u, v)=\frac{(u+v)((2 p+\sqrt{4 p+5}+3) u-2(p+1) v)}{2(p+1) \sqrt{4 p+5}(p+u+v+1)}, \\
\tilde{g}_{1}(u, v)=\frac{(u+v)(2(p+1) u+(-2 p+\sqrt{4 p+5}-3) v)}{2(p+1) \sqrt{4 p+5}(p+u+v+1)} .
\end{gathered}
$$

3.2. Unstable manifold of the equilibrium solution $\bar{x}$. Let us assume that $0<p<1$ and that the local unstable manifold corresponding to the saddle point $(\bar{x}, \bar{x})$ is the graph of the map $\varphi$ of the form

$$
\varphi(\xi)=\alpha \xi^{2}+\beta \xi^{3}+O\left(|\xi|^{4}\right), \quad \alpha, \beta \in \mathbb{R} .
$$

Now we compute the constants $\alpha$, and $\beta$

The function $\varphi$ must satisfy the unstable manifold equation

$$
\varphi\left(\mu_{1} \xi+\tilde{f}_{1}(\xi, \varphi(\xi))\right)=\mu_{2} \varphi(\xi)+\tilde{g}_{1}(\xi, \varphi(\xi)),
$$


which leads to the following polynomial equation

$$
p_{1} \xi^{2}+p_{2} \xi^{3}+\cdots+p_{17} \xi^{18}=0
$$

By solving the system $p_{1}=0$ and $p_{2}=0$, we obtain the values

$$
\begin{aligned}
& \alpha=-\frac{2(p+1)}{p(4 p-3 \sqrt{4 p+5}+5)-4 \sqrt{4 p+5}}, \\
& \beta=\frac{(p+1)(2 p(2 p+5)+\sqrt{4 p+5}+7)}{(4 p+5)\left(2 p^{4}+10 p^{3}+14 p^{2}+p-6-\sqrt{4 p+5}\left(2 p^{3}+6 p^{2}+9 p+6\right)\right)} .
\end{aligned}
$$

Since

$$
\eta_{n}=\alpha \xi_{n}^{2}+\beta \xi_{n}^{3} \quad \text { and } \quad\left(\begin{array}{c}
\xi_{n} \\
\eta_{n}
\end{array}\right)=P^{-1} \cdot\left(\begin{array}{c}
x_{n-1}-p-1 \\
x_{n}-p-1
\end{array}\right)
$$

we can approximate locally the local unstable manifold $\mathcal{W}_{\text {loc }}^{u}(\bar{x}, \bar{x})$ as the graph of the map $\tilde{\varphi}(x)$ such that $U(x, \tilde{\varphi}(x))=0$ where

$$
\begin{aligned}
& U(x, y):=\frac{2(-p+x-1)+(\sqrt{4 p+5}-1)(-p+y-1)}{2 \sqrt{4 p+5}} \\
& -\frac{(p+1)(2 p(2 p+5)+\sqrt{4 p+5}+7)}{8(4 p+5)^{5 / 2}\left(2 p^{4}+10 p^{3}+14 p^{2}+p-6-\sqrt{4 p+5}\left(2 p^{3}+6 p^{2}+9 p+6\right)\right)} \\
& \cdot(-2(-p+x-1)+\sqrt{4 p+5}(-p+y-1)-p+y-1)^{3} \\
& +\frac{(p+1)(-2(-p+x-1)+\sqrt{4 p+5}(-p+y-1)-p+y-1)^{2}}{2(4 p+5)(p(4 p-3 \sqrt{4 p+5}+5)-4 \sqrt{4 p+5})}
\end{aligned}
$$

and which satisfies

$$
\tilde{\varphi}(\bar{x})=\bar{x} \text { and } \tilde{\varphi}^{\prime}(\bar{x})=\frac{2}{1-\sqrt{4 p+5}} .
$$

Thus we proved the following result

Theorem 6. The local unstable manifold of Equation (1) corresponding to the saddle point $\bar{x}$ is given with the asymptotic expansion $U(x, \tilde{\varphi}(x))=0$.

3.3. Stable manifold of the equilibrium solution $\bar{x}$. Suppose $0<p<1$ and that the local stable manifold corresponding to the saddle point $\bar{x}=$ $p+1$, is the graph of the map $\psi$ that has the form

$$
\psi(\eta)=\gamma \eta^{2}+\delta \eta^{3}+O\left(|\eta|^{4}\right), \quad \gamma, \delta \in \mathbb{R} .
$$

Now we compute the constants $\gamma$ and $\delta$.

The function $\psi$ must satisfy the unstable manifold equation

$$
\psi\left(\mu_{2} \eta+\tilde{g}_{1}(\psi(\eta), \eta)\right)=\mu_{1} \psi(\eta)+\tilde{f}_{1}(\psi(\eta), \eta),
$$


which leads to the following polynomial equation

$$
q_{1} \eta^{2}+q_{2} \eta^{3}+\cdots+q_{17} \eta^{18}=0
$$

By solving system $q_{1}=0$ and $q_{2}=0$, we obtain the values

$$
\begin{aligned}
& \gamma=-\frac{2(p+1)}{p(4 p+3 \sqrt{4 p+5}+5)+4 \sqrt{4 p+5}}, \\
& \delta=\frac{(a+1)(2 p(2 p+5)-\sqrt{4 p+5}+7)}{(4 p+5)\left(2 p^{4}+10 p^{3}+14 p^{2}+p-6+\sqrt{4 p+5}\left(2 p^{3}+6 p^{2}+9 p+6\right)\right)} .
\end{aligned}
$$

Since

$$
\xi_{n}=\gamma \eta_{n}^{2}+\delta \eta_{n}^{3}, \quad\left(\begin{array}{c}
\xi_{n} \\
\eta_{n}
\end{array}\right)=P^{-1} \cdot\left(\begin{array}{c}
x_{n-1}-p-1 \\
x_{n}-p-1
\end{array}\right),
$$

we can approximate locally the local stable manifold $\mathcal{W}_{\text {loc }}^{s}(\bar{x}, \bar{x})$ as the graph of the map $\tilde{\psi}(y)$ such that $S(\tilde{\psi}(y), y)=0$, where

$$
\begin{aligned}
& S(x, y):= \frac{-2(-p+x-1)+\sqrt{4 p+5}(-p+y-1)-p+y-1}{2 \sqrt{4 p+5}} \\
&-\frac{(p+1)(2 p(2 p+5)-\sqrt{4 p+5}+7)}{8(4 p+5)^{5 / 2}\left(2 p^{4}+10 p^{3}+14 p^{2}+p-6+\sqrt{4 p+5}\left(2 p^{3}+6 p^{2}+9 p+6\right)\right)} \\
&+(2(-p+x-1)+(\sqrt{4 p+5}-1)(-p+y-1))^{3} \\
&+\frac{(p+1)(2(-p+x-1)+(\sqrt{4 p+5}-1)(-p+y-1))^{2}}{2(4 p+5)(p(4 p+3 \sqrt{4 p+5}+5)+4 \sqrt{4 p+5})}, \quad(20)
\end{aligned}
$$

which satisfies

$$
\tilde{\psi}_{1}(\bar{x})=\bar{x} \text { and } \tilde{\psi}^{\prime}(\bar{x})=\frac{2}{1+\sqrt{4 p+5}} .
$$

Thus we proved the following result

Theorem 7. The local stable manifold of Equation (1) corresponding to the saddle point $\bar{x}$ is locally given with the asymptotic expansion $S(\tilde{\psi}(y), y)=0$.

3.4. Some numerical examples of stable and unstable manifolds. In this section we provide some numerical examples and we compare visually the asymptotic approximations of stable and unstable manifolds, obtained by using Mathematica, with the boundaries of the basins of attraction obtained by using the software package Dynamica 4 [11].

For $p=0.2$ we have that

$$
\begin{aligned}
S^{1}(x, y)= & -0.000877571(2(x-1.2)+1.40832(y-1.2))^{3} \\
& +0.00845285(2(x-1.2)+1.40832(y-1.2))^{2} \\
& +0.207614(-2(x-1.2)+2.40832(y-1.2)+y-1.2),
\end{aligned}
$$




$$
\begin{aligned}
U^{1}(x, y)= & 0.000872157(-2(x-1.2)+2.40832(y-1.2)+y-1.2)^{3} \\
& -0.0104301(-2(x-1.2)+2.40832(y-1.2)+y-1.2)^{2} \\
& +0.207614(2(x-1.2)+1.40832(y-1.2)),
\end{aligned}
$$

and for $p=0.8$ we have that

$$
\begin{aligned}
S^{2}(x, y)= & -0.000279578(2(x-1.8)+1.86356(y-1.8))^{3} \\
& +0.00441021(2(x-1.8)+1.86356(y-1.8))^{2} \\
& +0.174608(-2(x-1.8)+2.86356(y-1.8)+y-1.8), \\
U^{2}(x, y)= & 0.000567856(-2(x-1.8)+2.86356(y-1.8)+y-1.8)^{3} \\
& -0.0093276(-2(x-1.8)+2.86356(y-1.8)+y-1.8)^{2} \\
& +0.174608(2(x-1.8)+1.86356(y-1.8)) .
\end{aligned}
$$

Figures 1 and 2 show the graph of the functions $S^{1}(x, y)=0, U^{1}(x, y)=0$ and $S^{2}(x, y)=0, U^{2}(x, y)=0$ with the basins of attraction created with Dynamica 4 .
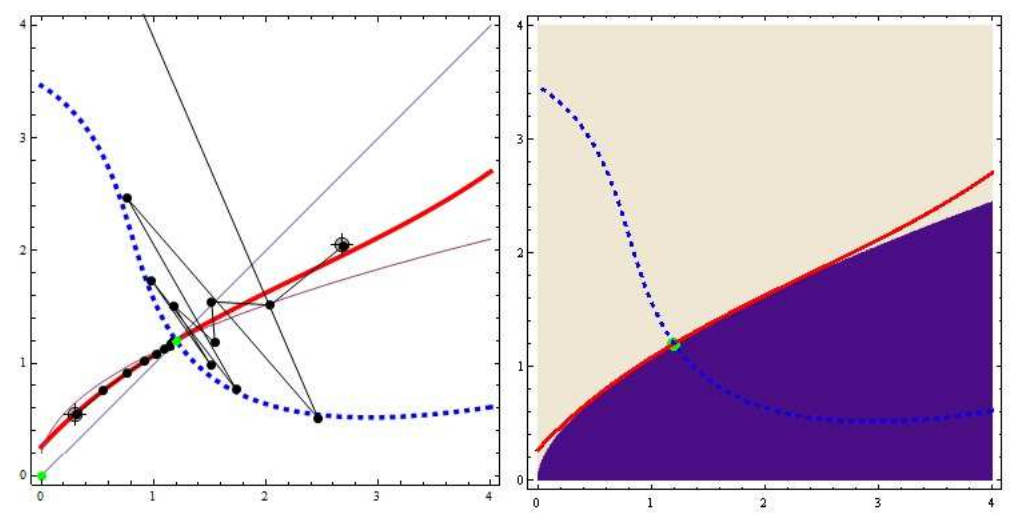

Figure 1. The graph of the function $S^{1}(x, y)=0$ (red curves) and $U^{1}(x, y)=0$ (blue curves) for $p=0.2$ with the basins of attraction generated by Dynamica 4 . The typical trajectory follows the unstable manifold.

3.5. Normal form of the map $T^{2}$ at the non-hyperbolic period-two solution $\{(\Phi, \Phi /(\Phi-1)),(\Phi /(\Phi-1), \Phi)\}$. If $p=1$ then Equation (1) has an infinite number of period-two solutions which are non-hyperbolic points of the stable type. If $\Phi>1$ then it is easy to see that all period-two 


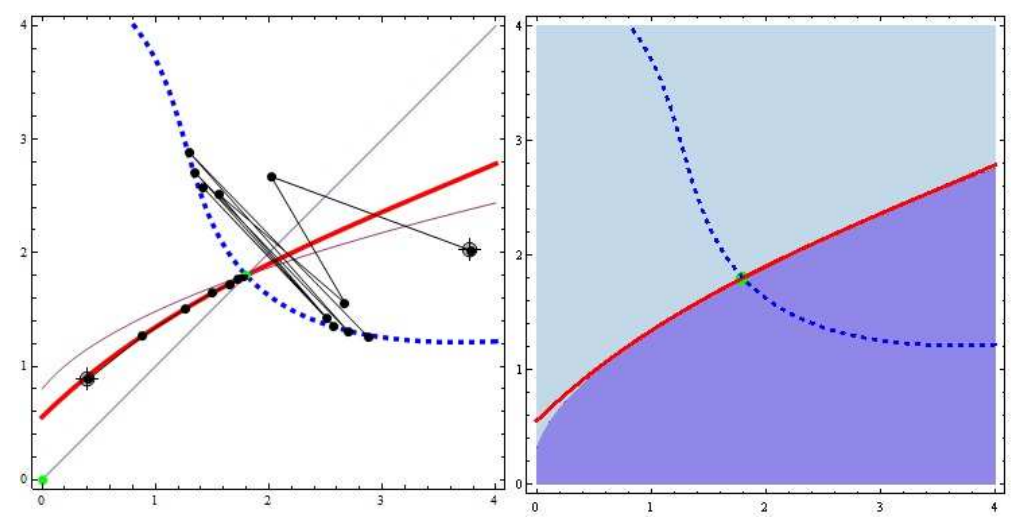

Figure 2. The graph of the function $S^{2}(x, y)=0$ (red curves) and $U^{2}(x, y)=0$ (blue curves) for $p=0.8$ with the basins of attraction generated by Dynamica 4 . The typical trajectory follows the unstable manifold.

solutions are given by $(\Phi, \Phi /(\Phi-1))$. First we transform the period-two solution $(\Phi, \Phi /(\Phi-1))$ of (1) to the origin by translation

$$
\tilde{u}=u-\Phi \quad \text { and } \quad \tilde{v}=v-\frac{\Phi}{\Phi-1}
$$

under which the corresponding map (13) becomes

$$
\left(\begin{array}{c}
\tilde{u} \\
\tilde{v}
\end{array}\right) \rightarrow \tilde{F}\left(\begin{array}{c}
\tilde{u} \\
\tilde{v}
\end{array}\right)=T^{2}\left(\begin{array}{c}
\tilde{u}+\Phi \\
\tilde{v}+\frac{\Phi}{\Phi-1}
\end{array}\right)-\left(\begin{array}{c}
\Phi \\
\Phi \\
\Phi-1
\end{array}\right)=\left(\begin{array}{c}
\frac{(\Phi-1)(\tilde{u}-\Phi \tilde{v}+\tilde{v})}{(\Phi-1) \tilde{v}+\Phi} \\
-\frac{\tilde{u}+\tilde{v}(-\Phi(\tilde{v}+2)+\tilde{v}+1)}{(\Phi-1) \tilde{u}+(\Phi-1) \tilde{v}+\Phi^{2}}
\end{array}\right) .
$$

Then $\tilde{F}$ has the fixed point at $(0,0)$. The Jacobian matrix of $\tilde{F}$ at $(0,0)$ has the form

$$
J_{0}=J a c_{\tilde{F}}(0,0)=\left(\begin{array}{cc}
\frac{\Phi-1}{\Phi} & -\frac{(\Phi-1)^{2}}{\Phi} \\
-\frac{1}{\Phi^{2}} & \frac{2 \Phi-1}{\Phi^{2}}
\end{array}\right) .
$$

The eigenvalues of (22) are

$$
\mu_{1}=\frac{\Phi-1}{\Phi^{2}}<1 \quad \text { and } \quad \mu_{2}=1,
$$

which shows that $(\Phi, \Phi /(\Phi-1))$ is the fixed point of stable type. The eigenvectors corresponding to the eigenvalues $\mu_{1,2}$ are given by

$$
\mathbf{v}_{1}=(\Phi, 1)^{T} \text { and } \mathbf{v}_{2}=\left(-(\Phi-1)^{2}, 1\right)^{T},
$$

respectively. 
Then we have that

$$
\left(\begin{array}{l}
\tilde{u} \\
\tilde{v}
\end{array}\right) \rightarrow \tilde{F}\left(\begin{array}{c}
\tilde{u} \\
\tilde{v}
\end{array}\right)=\left(\begin{array}{cc}
\frac{\Phi-1}{\Phi} & -\frac{(\Phi-1)^{2}}{\Phi} \\
-\frac{1}{\Phi^{2}} & \frac{2 \Phi-1}{\Phi^{2}}
\end{array}\right)\left(\begin{array}{l}
\tilde{u} \\
\tilde{v}
\end{array}\right)+\tilde{H}_{1}\left(\begin{array}{c}
\tilde{u} \\
\tilde{v}
\end{array}\right)
$$

where

$$
\tilde{H}\left(\begin{array}{c}
\tilde{u} \\
\tilde{v}
\end{array}\right):=\left(\begin{array}{c}
-\frac{(\Phi-1)^{2} \tilde{v}(\tilde{u}-\Phi \tilde{v}+\tilde{v})}{\Phi((\Phi-1) \tilde{v}+\Phi)} \\
\frac{(\Phi-1)(\tilde{u}-\Phi \tilde{v}+\tilde{v})^{2}}{\Phi^{2}\left((\Phi-1) \tilde{u}+(\Phi-1) \tilde{v}+\Phi^{2}\right)}
\end{array}\right)
$$

Let

$$
\left(\begin{array}{c}
\tilde{u} \\
\tilde{v}
\end{array}\right)=P \cdot\left(\begin{array}{l}
\xi \\
\eta
\end{array}\right) \text { where } P=\left(\mathbf{v}_{1}, \mathbf{v}_{2}\right)=\left(\begin{array}{cc}
\Phi & -(\Phi-1)^{2} \\
1 & 1
\end{array}\right) .
$$

Then (23) leads to the corresponding normal form

$$
\left(\begin{array}{l}
\xi \\
\eta
\end{array}\right) \rightarrow\left(\begin{array}{cc}
\mu_{1} & 0 \\
0 & \mu_{2}
\end{array}\right)\left(\begin{array}{l}
\xi \\
\eta
\end{array}\right)+\left(\begin{array}{c}
\tilde{f}_{2}(\xi, \eta) \\
\tilde{f}_{2}(\xi, \eta)
\end{array}\right)
$$

where

$$
\left(\begin{array}{c}
\tilde{f}_{2}(\xi, \eta) \\
\tilde{g}_{2}(\xi, \eta)
\end{array}\right):=P^{-1} \cdot \widetilde{H}_{1}\left(P \cdot\left(\begin{array}{l}
\xi \\
\eta
\end{array}\right)\right)
$$

One can see that

$$
\left(\begin{array}{l}
\tilde{f}_{2}(\xi, \eta) \\
\tilde{g}_{2}(\xi, \eta)
\end{array}\right)=\left(\begin{array}{c}
-\frac{(\Phi-1)^{2}(\xi-\eta(\Phi-1) \Phi)\left(\frac{(\Phi-1)(\xi-\eta(\Phi-1) \Phi)}{-\Phi^{2}(3 \eta+\xi+1)+\eta \Phi^{3}+2 \eta \Phi+\xi}+\frac{\Phi(\eta+\xi)}{\eta(\Phi-1)+\xi(\Phi-1)+\Phi}\right)}{\Phi^{2}((\Phi-1) \Phi+1)} \\
\frac{(\Phi-1)(\xi-\eta(\Phi-1) \Phi)\left(\frac{(\Phi-1)(\eta+\xi)}{\eta(\Phi-1) \xi(\Phi-1)+\Phi}-\frac{\xi-\eta(\Phi-1) \Phi}{-\Phi^{2}(3 \eta+\xi+1)+\eta \Phi^{3}+2 \eta \Phi+\xi}\right)}{\Phi((\Phi-1) \Phi+1)}
\end{array}\right) .
$$

3.6. Stable set of period-two solution $\{(\Phi, \Phi /(\Phi-1)),(\Phi /(\Phi-1), \Phi)\}$. Let us assume that the invariant curve $\mathcal{C}$ corresponding to a non-hyperbolic point $(\Phi, \Phi /(\Phi-1))$ is the graph of the map $\varphi$ of the form

$$
h(\xi)=\tilde{\alpha} \xi^{2}+\tilde{\beta} \xi^{3}+O\left(|\xi|^{4}\right), \quad \tilde{\alpha}, \tilde{\beta} \in \mathbb{R} .
$$

Now we compute the constants $\tilde{\alpha}$, and $\tilde{\beta}$

The function $h$ must satisfy the equation

$$
h\left(\mu_{1} \xi+\tilde{f}_{2}(\xi, h(\xi))\right)=h(\xi)+\tilde{g}_{2}(\xi, h(\xi)),
$$

which leads to the following polynomial equation

$$
p_{1} \xi^{2}+p_{2} \xi^{3}+\cdots+p_{17} \xi^{18}=0 .
$$


By solving system $p_{1}=0$ and $p_{2}=0$, we obtain the values

$$
\begin{aligned}
& \tilde{\alpha}=-\frac{(\Phi-1) \Phi}{\Phi\left(\Phi^{3}-\Phi+2\right)-1}, \\
& \tilde{\beta}=\frac{(\Phi-1)^{2} \Phi(\Phi(\Phi((\Phi-1) \Phi+5)-4)+1)}{((\Phi-1) \Phi+1)^{2}\left(\Phi^{2}+\Phi-1\right)\left(\Phi^{4}+\Phi^{3}-2 \Phi+1\right)} .
\end{aligned}
$$

Since

$$
\eta_{2 n}=\tilde{\alpha} \xi_{2 n}^{2}+\tilde{\beta} \xi_{2 n}^{3}, \quad \text { and } \quad\left(\begin{array}{c}
\xi_{2 n} \\
\eta_{2 n}
\end{array}\right)=P^{-1} \cdot\left(\begin{array}{c}
x_{2 n-2}-\Phi \\
x_{2 n}-\frac{\Phi}{\Phi-1}
\end{array}\right)
$$

we can approximate locally invariant curve $\mathcal{C}$ as the graph of the map $\tilde{h}(x)$ such that $C(x, \tilde{h}(x))=0$ where

$$
\begin{gathered}
C(x, y ; \Phi):=\frac{(\Phi-1) \Phi\left(-\Phi+x+(\Phi-1)^{2}\left(y-\frac{\Phi}{\Phi-1}\right)\right)^{2}}{((\Phi-1) \Phi+1)^{2}\left(\Phi\left(\Phi^{3}-\Phi+2\right)-1\right)}- \\
\frac{(\Phi-1)^{2} \Phi(\Phi(\Phi((\Phi-1) \Phi+5)-4)+1)\left(-\Phi+x+(\Phi-1)^{2}\left(y-\frac{\Phi}{\Phi-1}\right)\right)^{3}}{((\Phi-1) \Phi+1)^{5}\left(\Phi^{2}+\Phi-1\right)\left(\Phi^{4}+\Phi^{3}-2 \Phi+1\right)} \\
+\frac{\Phi-x+\Phi\left(y-\frac{\Phi}{\Phi-1}\right)}{(\Phi-1) \Phi+1},
\end{gathered}
$$

which satisfies

$$
\tilde{h}(\Phi)=\frac{\Phi}{\Phi-1} \text { and } \tilde{h}^{\prime}(\Phi)=\frac{1}{\Phi} .
$$

Thus we proved the following result

Theorem 8. Assume that $\Phi>1$. The invariant curve $\mathcal{C}$ corresponding to the non-hyperbolic period-two solution $\{(\Phi, \Phi /(\Phi-1)),(\Phi /(\Phi-1), \Phi)\}$ is the union of two curves which are locally given with the asymptotic expansions $C(x, \tilde{h}(x) ; \Phi)=0$ and $C(x, \tilde{h}(x) ; \Phi /(\Phi-1))=0$.

3.7. Some numerical examples for the invariant curve $\mathcal{C}$. In this section we provide some numerical examples for invariant curve $C$, obtained by using Mathematica.

For $\Phi=2.94$ we have that

$$
\begin{aligned}
C_{1}(x, y ; \Phi)= & -0.000066293(x+3.7636(y-1.51546)-2.94)^{3} \\
& +0.00178892(x+3.7636(y-1.51546)-2.94)^{2} \\
& +0.149174(-x+2.94(y-1.51546)+2.94),
\end{aligned}
$$




$$
\begin{aligned}
C_{1}(x, y ; \Phi /(\Phi-1))= & -0.00975853(x+0.265703(y-2.94)-1.51546)^{3} \\
& +0.0491587(x+0.265703(y-2.94)-1.51546)^{2} \\
& +0.56143(-x+1.51546(y-2.94)+1.51546),
\end{aligned}
$$

and for $\Phi=2.3$ we have that

$$
\begin{aligned}
C_{2}(x, y ; \Phi)= & -0.000543625(x+1.69(y-1.76923)-2.3)^{3} \\
+ & 0.00714278(x+1.69(y-1.76923)-2.3)^{2} \\
+ & 0.250627(-x+2.3(y-1.76923)+2.3), \\
C_{2}(x, y ; \Phi /(\Phi-1))= & -0.00395629(x+0.591716(y-2.3)-1.76923)^{3} \\
& +0.0265206(x+0.591716(y-2.3)-1.76923)^{2} \\
& +0.423559(-x+1.76923(y-2.3)+1.76923) .
\end{aligned}
$$

Figures 3 show the graph of the functions $C_{1}(x, y ; 2.94)=0, C_{1}(x, y ; 1.51546)$ $=0$ and $C_{2}(x, y ; 2.3)=0, C_{2}(x, y ; 1.76923)=0$. One can see that the typical trajectory follows the invariant curve.
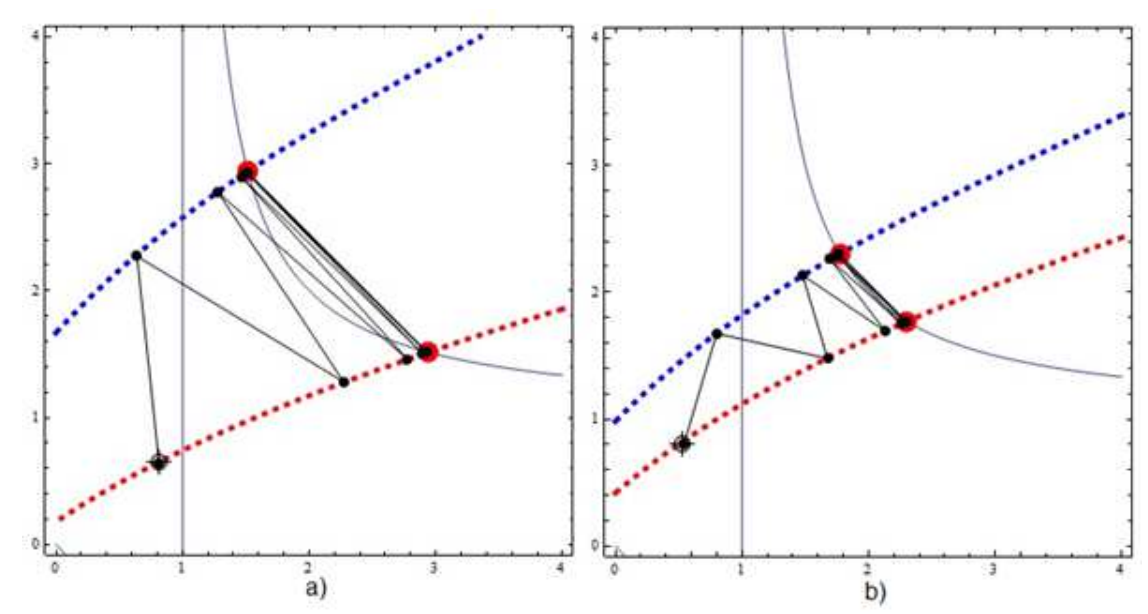

Figure 3. a) The graphs of the functions $C_{1}(x, y ; 2.94)=$ 0 (blue curve), $C_{1}(x, y ; 1.51546)=0$ (red curve) b) The graphs of the functions $C_{2}(x, y ; 2.3)=0$ (blue curve), $C_{2}(x, y ; 1.76923)=0$ (red curve). 


\section{REFERENCES}

[1] A. M. Amleh, E. A. Grove, D. A. Georgiou and G. Ladas, On the recursive sequence $x_{n+1}=\alpha+\frac{x_{n-1}}{x_{n}}$, , J. Math. Anal. Appl., 233 (1999), 790-798.

[2] J. Bektešević, M. R. S. Kulenović and E. Pilav, Asymptotic approximations of a stable and unstable manifolds of a two-dimensional quadratic map, J. Comp. Anal. Appl., 21 (2016), 35-51.

[3] E. Camouzis and G. Ladas, When does local asymptotic stability imply global attractivity in rational equations?, J. Difference Equ.Appl., 12 (2006), 863-885.

[4] D. Clark and M. R. S. Kulenović, A coupled system of rational difference equations, Comput. Math. Appl., 43 (2002), 849-867.

[5] A. Brett and M. R. S. Kulenović, Basins of attraction of equilibrium points of monotone difference equations, Sarajevo J. Math., 5 (2009), 211-233.

[6] Dž. Burgić, S. Kalabušić and M. R. S. Kulenović, Non-hyperbolic dynamics for competitive systems in the plane and global period-doubling bifurcations, Adv. Dyn. Syst. Appl., 3 (2008), 229-249.

[7] S. Elaydi, Discrete Chaos. With Applications in Science and Engineering, Second edition, With a foreword by Robert M. May, Chapman \& Hall/CRC, Boca Raton, FL, 2008.

[8] M. Guzowska, R. Luis and S. Elaydi, Bifurcation and invariant manifolds of the logistic competition model, J. Difference Equ. Appl., 17:12 (2011), 1851-1872.

[9] J. K. Hale and H. Kocak, Dynamics and Bifurcations, Texts in Applied Mathematics, 3. Springer-Verlag, New York, 1991.

[10] M. R. S. Kulenović and G. Ladas, Dynamics of Second Order Rational Difference Equations, Open Problems and Conjectures, Chapman\& Hall/CRC Press, Boca Raton, 2001.

[11] M. R. S. Kulenović and O. Merino, Discrete Dynamical Systems and Difference Equations with Mathematica, Chapman and Hall/CRC, Boca Raton, London, 2002.

[12] M. R. S. Kulenović and O. Merino, Competitive-exclusion versus competitivecoexistence for systems in the plane, Discrete Contin. Dyn. Syst. Ser. B, 6 (2006), 1141-1156.

[13] M. R. S. Kulenović and O. Merino, Convergence to a Period-Two Solution for a Class of Second Order Rational Difference Equations, Difference equations, special functions and orthogonal polynomials, Proc. Tenth International Conf. Difference Equations, Munich(Germany), World Sci. Publ., Hackensack, NJ, 2007., (2007), 344-353.

[14] M. R. S. Kulenović and O. Merino, Global bifurcation for competitive systems in the plane, Discrete Contin. Dyn. Syst. B, 12 (2009), 133-149.

[15] M. R. S. Kulenović and O. Merino, Invariant manifolds for competitive discrete systems in the plane, Internat. J. Bifur. Chaos Appl. Sci. Eng., 20 (8) (2010), 2471-2486.

[16] C. Robinson, Stability, Symbolic Dynamics, and Chaos, CRC Press, Boca Raton, 1995.

[17] H. L. Smith, Planar competitive and cooperative difference equations, J. Difference Equ. Appl., 3 (1998), 335-357.

[18] E. S. Thomas, A. Clark, and D. R. Wilken, A note on the geometry of invariants, J. Difference Equ. Appl., 12 (2006), 859-861.

[19] S. Wiggins, Introduction to Applied Nonlinear Dynamical Systems and Chaos, Second edition, Texts in Applied Mathematics, 2. Springer-Verlag, New York, 2003.

[20] D. R. Wilken, E. S. Thomas, and A. S. Clark, A proof of the no rational invariant theorem, J. Difference Equ. Appl., 12 (2006), 669-675. 
(Received: March 23, 2016)

M.R.S. Kulenović

Department of Mathematics

University of Rhode Island

Kingston, Rhode Island 02881-0816

U.S.A.

mkulenovic@uri.edu

E. Pilav

Department of Mathematics

University of Sarajevo

71000 Sarajevo

Bosnia and Herzegovina 